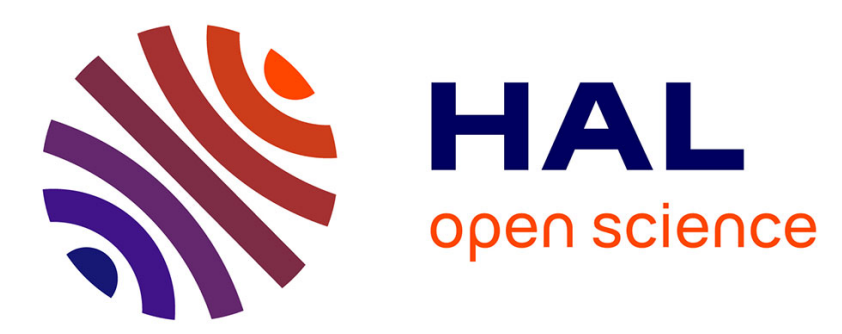

\title{
High fidelity closed loop controlled friction in SMARTTAC tactile stimulator
}

Wael Ben Messaoud, Michel Amberg, Betty Lemaire-Semail, Frédéric Giraud, Marie-Ange Bueno

\section{- To cite this version:}

Wael Ben Messaoud, Michel Amberg, Betty Lemaire-Semail, Frédéric Giraud, Marie-Ange Bueno. High fidelity closed loop controlled friction in SMARTTAC tactile stimulator. 2015 17th European Conference on Power Electronics and Applications (EPE'15 ECCE-Europe), Sep 2015, Geneva, Switzerland. 10.1109/EPE.2015.7309191 . hal-01252057

\section{HAL Id: hal-01252057 \\ https://inria.hal.science/hal-01252057}

Submitted on 7 Jan 2016

HAL is a multi-disciplinary open access archive for the deposit and dissemination of scientific research documents, whether they are published or not. The documents may come from teaching and research institutions in France or abroad, or from public or private research centers.
L'archive ouverte pluridisciplinaire HAL, est destinée au dépôt et à la diffusion de documents scientifiques de niveau recherche, publiés ou non, émanant des établissements d'enseignement et de recherche français ou étrangers, des laboratoires publics ou privés. 


\title{
High fidelity closed loop controlled friction in SMARTTAC tactile stimulator
}

\author{
Wael Ben Messaoud ${ }^{1,2}$, Michel Amberg ${ }^{1}$, Betty Lemaire-Semail ${ }^{1}$ \\ Frédéric Giraud $^{1}$ and Marie-Ange Bueno ${ }^{2}$ \\ ${ }^{1}$ Laboratoire d'Electrotechnique et d'Electronique de Puissance \\ University Lille 1, 50, Avenue Halley. 59650 Villeneuve d'Ascq, France \\ wael.ben-messaoud@ed.univ-lille1.fr \\ ${ }^{2}$ Laboratoire de Physique et Mécanique Textiles \\ University of Haute Alsace, 11, rue Alfred Werner, 68093 Mulhouse, France
}

\section{Acknowledgements}

The authors gratefully acknowledge financial support from the Nord Pas de Calais and Alsace regions. Special thanks to IRCICA and INRIA institutions for supporting this work.

\section{Keywords}

«Tactile feedback», «Force Control», «Closed-Loop Control», «Sliding mode control», «Piezo actuators»

\begin{abstract}
The present study investigates the implementation of a closed loop control in a new smart tactile stimulator called "SMARTTAC". This haptic device is able to stimulate the fingertip in order to simulate the texture perception of different real surfaces such as gratings. Two closed loop control are implemented to ensure both a stable vibration amplitude despite of external disturbances and accurate control of the friction coefficient using the new design of the tactile stimulator. Experimental measurements give satisfactory results both on dynamics and robustness points of view.
\end{abstract}

\section{Introduction}

Nowadays, the sense of touch has been increasingly explored by the design of tactile devices aiming to render the sensation of touch of real surfaces using a programmable device. Different characteristics of the surface can be reproduced in the experience of touch, for example temperature, softness or texture friction characteristics. Three major types of friction modulation devices can be classified depending on the operating technology to produce the tactile sensation. The surface acoustic wave is a technology able to change the fineness of the surface's grain [1], the electrovibration technology increases the friction by creating an attractive electrostatic force between the sliding finger and the surface [2], [3] and the ultrasonic friction-modulation devices operates to reduce the friction by creating an air film lubricating the contact finger and the surface [4], [5].

The new tactile stimulator called "SMARTTAC" is a device able to stimulate the fingertip exploring its surface in order to simulate different textures. This device is an ultrasonic friction modulation device because it is based on producing ultrasonic vibration of a plate above which the finger is sliding. The objective is to use this device for fabrics simulation, particularly for the e-commerce shopping. One of the significant difference between reality and virtual shopping is that the purchaser cannot have the opportunity to touch and compare products [6]. In that case, a virtual tactile feedback provided by a tactile stimulator could be helpful. But to be able to simulate such complex textures, the tactile feedback device must be accurate and reliable. So the aim of this study is to propose a control strategy in order to cope with the numerous phenomena which may disturb the touch feedback. Such a stimulator could be used also to help people who suffer from tactile disorders for diagnosis and rehabilitation processes. On a technical point of view, this tactile stimulator is based on the creation of 
an air gap between the finger and a flat vibrating plate, thanks to a high frequency, low amplitude vibration of the surface. This vibration is able to reduce the friction coefficient [4], [7], [8]. In order to fulfil the requirement of high fidelity simulation, the device is equipped with closed control loops assuming the vibration amplitude control as well as the tuning of the operating frequency; this allows to cope with external disturbances during the exploration process, while keeping accurate dynamic and static performances, and reduction of energy consumption as well [9]. Moreover, SMARTTAC can measure the friction and normal forces during tactile task, as a tribometer. The advantage in comparison to an external tribometer is that it is possible to control the vibration, as a function of the finger-to-stimulator friction.

In this paper, the new tactile stimulator will be presented, then, the modelling and identifying process will be exposed before proceeding to the control of the system. Finally, experimental tries of the controlled system will be carried out showing on one side the accuracy of the vibration amplitude control and on the other side, the first attempts for actual friction regulation.

\section{Description of the "SMARTTAC" stimulator}

The SMARTTAC is a developed version of the STIMTAC stimulator [10]; it is actuated by piezoelectric ceramics to make a plate vibrating at one of its ultrasonic resonant frequency. Eight piezoceramics (four in each extremity) are glued under the active surface of the aluminum plate; the stimulator is sized $76 \times 41 \times 1.2 \mathrm{~mm}^{3}$ (Fig. 1a). In comparison with STIMTAC, "SMARTTAC" is a smart tactile stimulator because it integrates new sensors allowing a real time measurement of the lateral force between the fingertip and the vibrating plate during the exploration process. For the lateral force measurement, two strain gauge sensors (S1) and (S2) (FSS1500 from Honeywell) are placed in a compact and deformable mechanical structure, especially designed for this application, placed under the plate (Fig. 1b). S1 and S2 measure respectively the forces in $\mathrm{x}$ and y directions. After calibration the tangential force applied on the plate is obtained. The mechanical structure is composed by a support frame (A), tightened by two screws to the top and bottom of the tactile plate, and elastically connected to a central part (C) attached by two more screws to a solid aluminum base (D). This elasticity is allowed thanks to six deformable slats (B) between (A) and (C) when the finger is sliding. The normal force applied by the finger is measured from four other strain gauges located at the vibration nodes (S3-S6). The friction coefficient (COF) can be easily deduced thanks to Coulomb's law on each sampling period. To sum up, the "SMARTTAC" is the unique device which combines a stimulator based on the friction reduction and a tribometer.

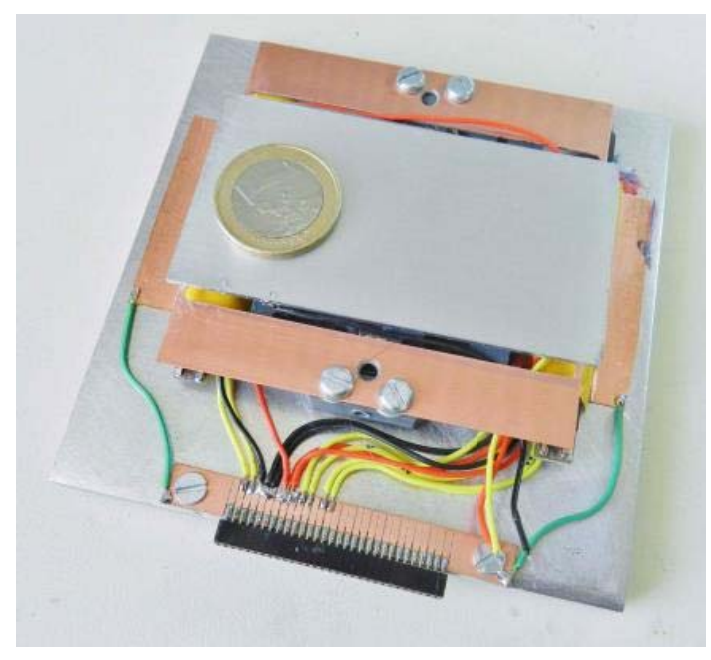

(a)

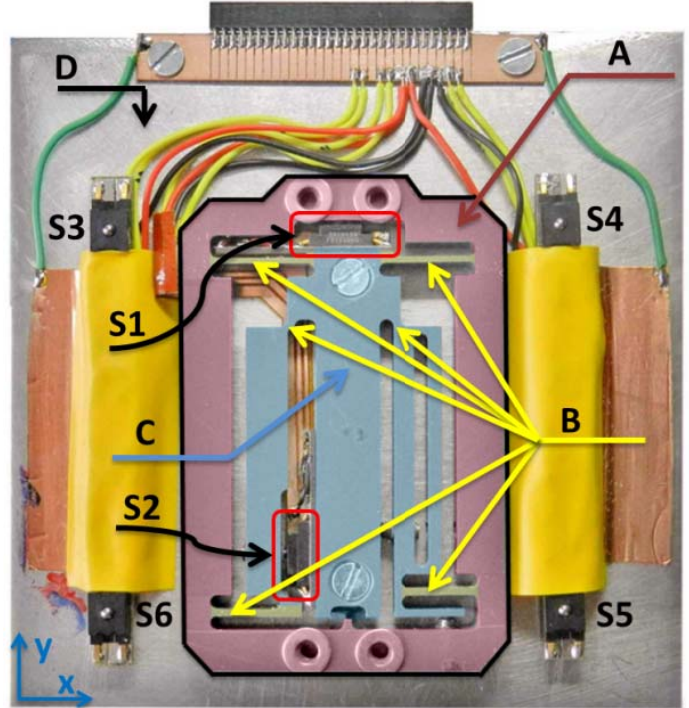

(b)

Fig. 1 a): Photo of the SMARTTAC, b): Fabricated mechanical structure placed under the active vibrating plate, able to measure using two force sensors, the lateral force applied by the fingertip when it explores the active surface of "SMARTTAC" 


\section{Vibration amplitude modelling}

In this section, we propose a modelling of the vibration amplitude behaviour to compensate the disturbance factors influencing on the vibration of the stimulator such as the influence of the finger pressure and the influence of the resonant frequency due to the variation of temperature [9], [11]. So, the system can be seen as Multiple-Input Multiple-Output or MIMO. The two inputs are the components of the voltage supply, which are the voltage amplitude $V$ and the instantaneous excitation angular frequency $\omega$. In other words, the system can be represented in a rotating d-q frame, with two components $V_{d}$ and $V_{q}$; this representation is classical for the study of electromagnetic machines [12] and it was also operated in the case of a standing wave piezoelectric actuator in [13]. The two outputs are the two components of the vibration amplitude which are the real and the imaginary parts respectively $W_{d}$ and $W_{q}$. The model of the tactile plate associated with a vibration mode is given by the following second order equation [13]:

$$
m \underline{\ddot{W}}+d_{s} \underline{\dot{W}}+c \underline{W}=N \underline{V}-F
$$

where $m$ is the modal mass, $d_{s}$ is the modal damping, $N$ is the electromechanical conversion factor and $c$ represents the modal stiffness. The vibration amplitude $W$ and the voltage $V$ can be represented with their complex form as following (the d-q axes are not yet defined):

$$
\begin{aligned}
& \underline{W}=\left(W_{d}+j W_{q}\right) e^{j \omega t} \\
& \underline{V}=\left(V_{d}+j V_{q}\right) e^{j \omega t}
\end{aligned}
$$

To simplify the modelling and the control, we choose the d-q frame attached to the voltage supply vector, such as $V_{d}=0$. By replacing the complex format of $W$ and $V$ (2) and (3) in (1), the real and imaginary parts of the equation (1) can be written as follows:

$$
\begin{aligned}
& m \ddot{W}_{d}+d_{s} \dot{W}_{d}+\left(c-m \omega^{2}\right) W_{d}-\omega\left(2 m \dot{W}_{q}+d_{s} W_{q}\right)=0 \\
& m \ddot{W}_{q}+d_{s} \dot{W}_{q}+\left(c-m \omega^{2}\right) W_{q}+\omega\left(2 m \dot{W}_{d}+d_{s} W_{d}\right)=N V_{q}
\end{aligned}
$$

At the resonance, $W_{q}=0$ because a $90^{\circ}$ is between the vector $\underline{V}$ and $\underline{W}$ [14].The modelling process of the system of equation (5) gives the transfer function between $W_{d}$ and $V$ which is a first order transfer function represented as follows:

$$
\frac{W_{d}(p)}{V(p)}=\frac{N / d_{s} \omega_{0}}{1+\left(2 m / d_{s}\right) p}
$$

with $\omega_{0}$ the angular resonant frequency. At vicinity of the resonance, if we control the $W_{d}$ based on the previous equation (6) so as to maintain it constant, their derivative terms of $W_{d}$ are zero. So, we extract from the equation (4) the model between $W_{q}(p) / W_{d}$ as a function of the variation of the angular frequency $\Delta \omega$ by considering $\omega=\omega_{0}+\Delta \omega$, synthetized as follows:

$$
\frac{\left(\frac{W_{q}(p)}{W_{d}}\right)}{\Delta \omega(p)}=\frac{\left(-2 m / d_{s}\right)}{1+\left(2 m / d_{s}\right) p}
$$

From the two models, we observe that the constant time of the two first order equations is the same. It may be noted also that the gain of the transfer function (7) is equal to the negative value of the 
constant time. It is the major advantage of this frame choice to give such a simple modelling, easy to identify experimentally.

After the identification of the process modelling, a vector control of $\underline{W}$ has been performed, leading to a high dynamic performance system and an accurate robustness to face finger pressure and temperature variation disturbances. Experimental results are given later to assess to performances of the controllers.

Nevertheless, these performances do not guarantee the accurate stimulation of the user, which highly depends on the finger-plate interaction and may vary from one user to the other. Another additional control must then be performed.

\section{Friction coefficient control}

\section{Problematic}

After tribological measurement of the coefficient of friction (COF) with 5 subjects (aged from 26 to 28 , average of 27) at 5 controlled vibration amplitudes $[0 ; 0.5 ; 1 ; 1.5 ; 2] \mu \mathrm{m}$, it can be observed in Fig. 2 that the percentage of the ratio between the reduced friction $\mu_{1}$ and the initial COF $\mu_{0}$ is highly variable depending on each person and on the conditions of measurement (the moisture for example increases the friction [15], [16]). The initial friction represents the friction between the finger of the user and the surface when the vibration is switched off whereas the reduced friction is the friction when the vibration is switched on at different level from 0 to $2 \mu \mathrm{m}$ with a step of $0.5 \mu \mathrm{m}$.

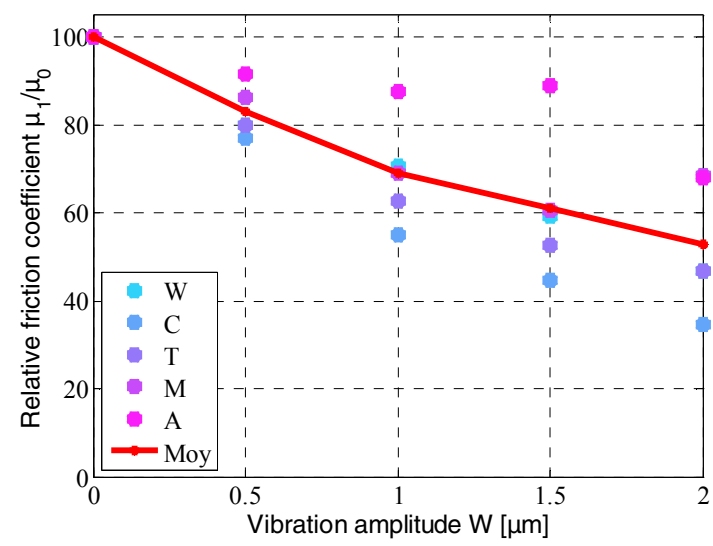

Fig. 2: The ratio between the reduced friction $\mu_{1}$ and the initial friction $\mu_{0}$ in $\%$ relative to the vibration amplitude for five subjects plotted by colored points. The mean of measurements of all subjects is plotted by a continuous red curve.

Our aim is to propose a tactile stimulator able to render accurately texture feelings whatever the touch conditions or the user finger characteristics. So, we propose a direct control of the COF in order to standardise the friction reduction which may be provided by the tactile feedback device. Using this smart stimulator called SMARTTAC described in the first part of this paper; it is possible to measure the friction and the normal forces leading to a real time measurement of the instantaneous friction coefficient $\mu=F t / F n$ between the finger and the surface of the plate. By knowing the instantaneous friction under the finger, an adjustment of the vibration amplitude can be achieved to impose a desired friction under the finger.

\section{Control method}

Because of the non-linear gain of the system having the vibration amplitude as input and the friction as output, it was not possible to implement a standard controller. So, we develop a controller based on data measurements. The sliding mode control is operated due its capability to operate with nonlinear systems [17]. It is placed in a closed loop including the previous vibration amplitude control (see Fig. 4). The controller acts on the vibration amplitude to control the friction coefficient between the finger 
and the plate. The reference of the controller is the difference between the instantaneous and the desired frictions divided by the desired friction coefficient as:

$$
E=100 * \frac{\mu_{1}-\mu_{1 \text { des }}}{\mu_{1 \text { des }}}
$$

The controller task is to minimise the parameter $E$ to impose the instantaneous desired friction. The controlled variable by the controller is the parameter $\Delta W$, it represents the value to add to the vibration amplitude $W$ to reach the friction reference. The developed controller operates at three cases; when $E \leq-25 \%$, the controller's output is $1 \mu \mathrm{m}$, if this ratio is up to $25 \%$, the controller output is $1 \mu \mathrm{m}$. the last case is when the ratio is between $-25 \%$ and $25 \%$, in this case the controller evolves according to the equation $W=0.04 * E$. The controller output is limited by a saturation set at $\pm 1 \mu \mathrm{m}$ to avoid a possible peak of vibration under the finger. The constant 0.04 multiplied by the parameter $E$ was chosen is order to find a compromise between the minimisation of the response time and the reduction of the chattering problem when the plate was controlled [18]. To sum up, the controller output is the following:

$$
\Delta W(E)=\left\{\begin{array}{cc}
1, & \text { if } \quad E \geq 25 \\
0.04 * E, & \text { if }-25<E<25 \\
-1, & \text { if } \quad E \leq-25
\end{array}\right.
$$

The figure 3 illustrates the controller output which is the vibration amplitude $W$ as a function of $\mathrm{E}$ which is the controller reference and can be considered as the friction error.

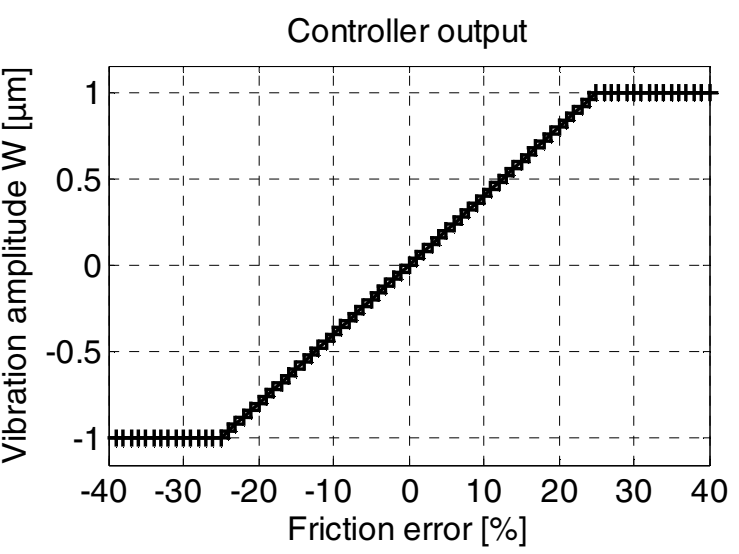

Fig. 3: Controller output acting on the vibration amplitude depending on the friction error $E$

To sum up, the closed loop control strategy method is based on two nested loops. The first one consists on controlling the vibration amplitude $W$ using the controller $C_{W}$ which acts on the voltage applied to actuators. The control method is based on modelling the system in a d-q frame to determine the transfer functions between inputs and outputs. The goal is to maintain $W$ constant and equal to the desired vibration amplitude whatever the disturbances and to vary the excitation frequency to guarantee the system operation at resonance. The main loop is based on a direct control of the friction between the sliding finger and the plate. The controller $C_{\mu}$ acts on the desired vibration $W_{\text {des }}$ to ensure a desired friction coefficient $\mu_{d e s}=\mu$. Fig 4 illustrates the operated closed loop control strategy. 


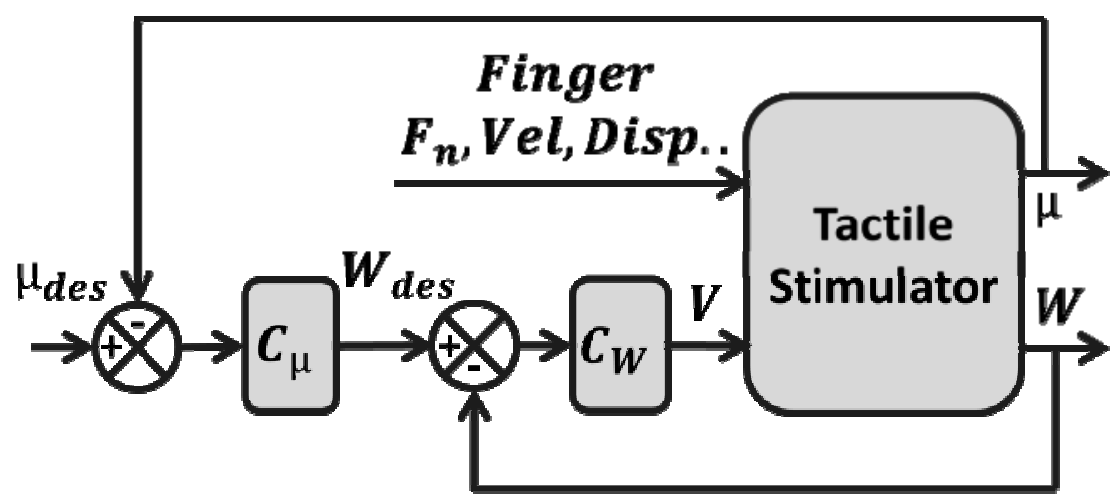

Fig. 4: The proposed control strategy aiming to a control of the vibration amplitude in the internal loop which is disturbed by the variation of the resonance frequency and the influence of the finger normal force, its velocity and displacement when the finger is sliding on the plate, while the main loop acts on the desired vibration amplitude to control the friction.

\section{Control implementation and experimental results}

This section aims at giving experimental results obtained first for the vibration amplitude control and second on the friction coefficient control.

\section{Vibration amplitude control}

Based on the identification of the two transfer functions, two PI controllers were calculated. The first controller adjusts the voltage in order to obtain a constant vibration amplitude (d axis), while the second controller is able to control the parameter $\Delta \omega$ to guarantee the excitation of the system at the resonance which maximizes the ratio vibration/voltage (q axis). The controllers are implemented together at $1 \mathrm{kHz}$ frequency real-time. The PI controller parameters are chosen to ensure both dynamic performances and robustness. As an example here, we have chosen to illustrate the robustness of the control law against the temperature variation: as a mechanical system, the tactile stimulator is sensitive to temperature variations which induce a shift in the resonant frequency. A variation of the resonant frequency is a real problem in use if it is not taken into account. In fact, the active surface is no longer excited at the resonance and the reduction of the friction is not at its maximum potential. These variations have been described in [3]. To check the robustness of our control law, the idea is to supply voltage at an initial frequency which corresponds to the resonant frequency at $30^{\circ} \mathrm{C}(43250 \mathrm{~Hz})$, although the real temperature during this experiment is about $20^{\circ} \mathrm{C}$ (that means a $0.3 \%$ higher resonant frequency). The closed loop should lead to a tracking of the actual resonance $(43390 \mathrm{~Hz})$ in a minimum time and as a consequence, the desired vibration amplitude will be obtained with less excitation voltage. Fig. 5 illustrates the fully controlled system behaviour with the two loops, in amplitude and angular frequency (red curve). The second result in Fig. 5 (green curve) corresponds to a second experiment for which the system is excited at the resonant frequency $43250 \mathrm{~Hz}\left(30^{\circ} \mathrm{C}\right.$ whereas we work at $20^{\circ} \mathrm{C}$ ), and only the vibration amplitude is controlled. The desired vibration amplitude for the two experiments was $1 \mu \mathrm{m}$ PtP. 


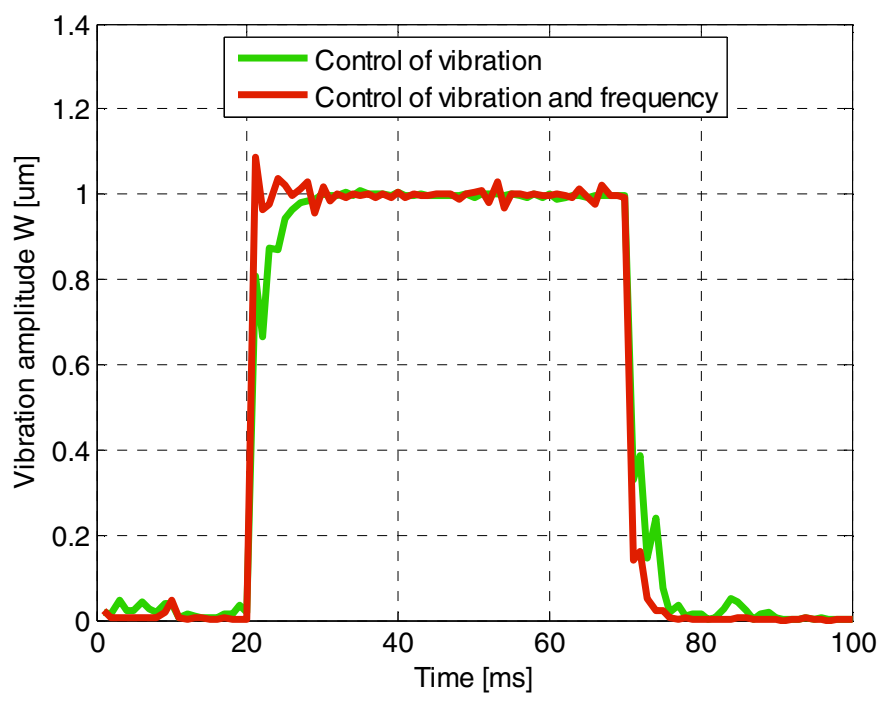

Fig. 5: Experimental comparison between vibration amplitude response of the controlled vibration system, and the response in the case of the double controlled system against temperature variation and vibration amplitude damping.

In static, it can be observed that in both experiments, the vibration amplitude is maintained constant $(1 \mu \mathrm{m})$. But, in dynamic, we can observe a difference in the $5 \%$ response time of the system $(5 \mathrm{~ms}$ in the vibration controlled system against $2 \mathrm{~ms}$ for the system controlled in vibration and frequency). To evaluate the importance of the double control in the energy consumption, Fig. 6 shows the excitation voltage required to reach $1 \mu \mathrm{m}$ of vibration amplitude PtP.

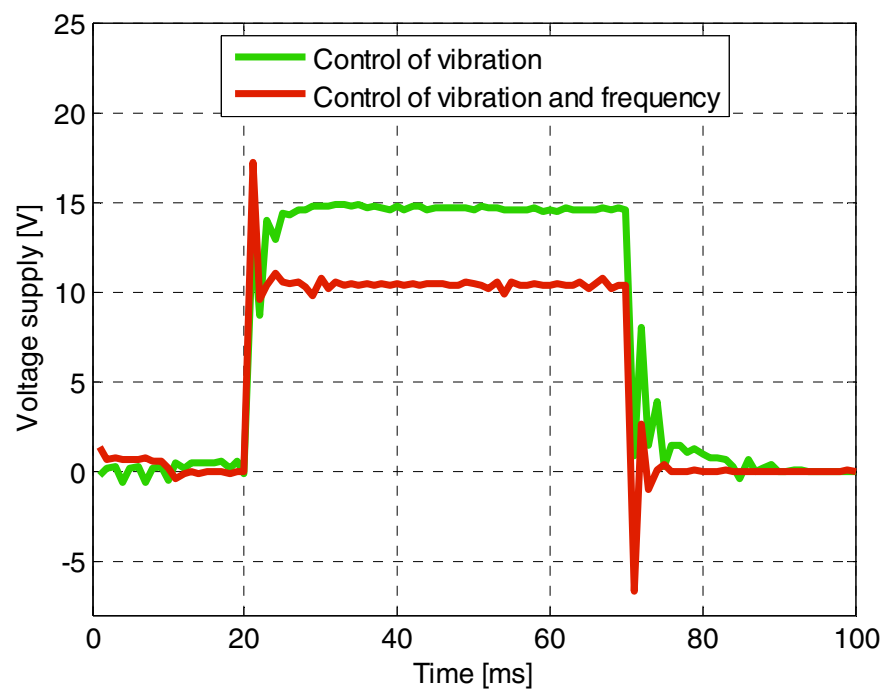

Fig. 6: Experimental comparison between voltage of the controlled vibration system, and the response in the case of the double controlled system against temperature variation and vibration amplitude damping.

In this figure, we can verify that the voltage supply is higher when the system is not operated at the resonance. The difference between the excitation voltages is about $30 \%$ which indicates the importance of the two controllers when implemented in the tactile stimulator. 


\section{Friction control results}

The previous control loops ensure the accuracy of the tactile stimulator itself on the point of view of physical variables such as vibration amplitude or energy consumption. However, the user has to be taken into account also on a tribological aspect. In this section, the interest is putted in the validation of the friction control strategy and its capability to impose a desired friction coefficient (COF) under the finger. The experiment consists on giving two references to the controller depending on the finger position. The difference between the two references in COF is imposed to be 0.1 in order to validate the controller robustness. Four subjects have been participated to this experiment, they were chosen according to their initial friction coefficient, which is different from a person to another, in order to test different operating points of the controller. The initial friction coefficient for the four subjects (ie without any vibration) is respectively between: 0.3 to $0.4,0.4$ to $0.5,0.5$ to 0.6 and 0.6 to 0.7 . The COF reference given to the controller depending on each subject is receptively: 0.2 to $0.3,0.3$ to 0.4 , 0.4 to 0.5 and 0.5 to 0.6 . Fig. 4 illustrates the controlled friction coefficient between a finger and the plate for each subject. The desired friction coefficient is between the two values as explained in the foregoing depending on the finger position. Fig. 7 illustrates the experimental results for all the subjects, the $\mathrm{x}$ label represents the time in $m s$ when the finger is altering between the two parts of the plate.
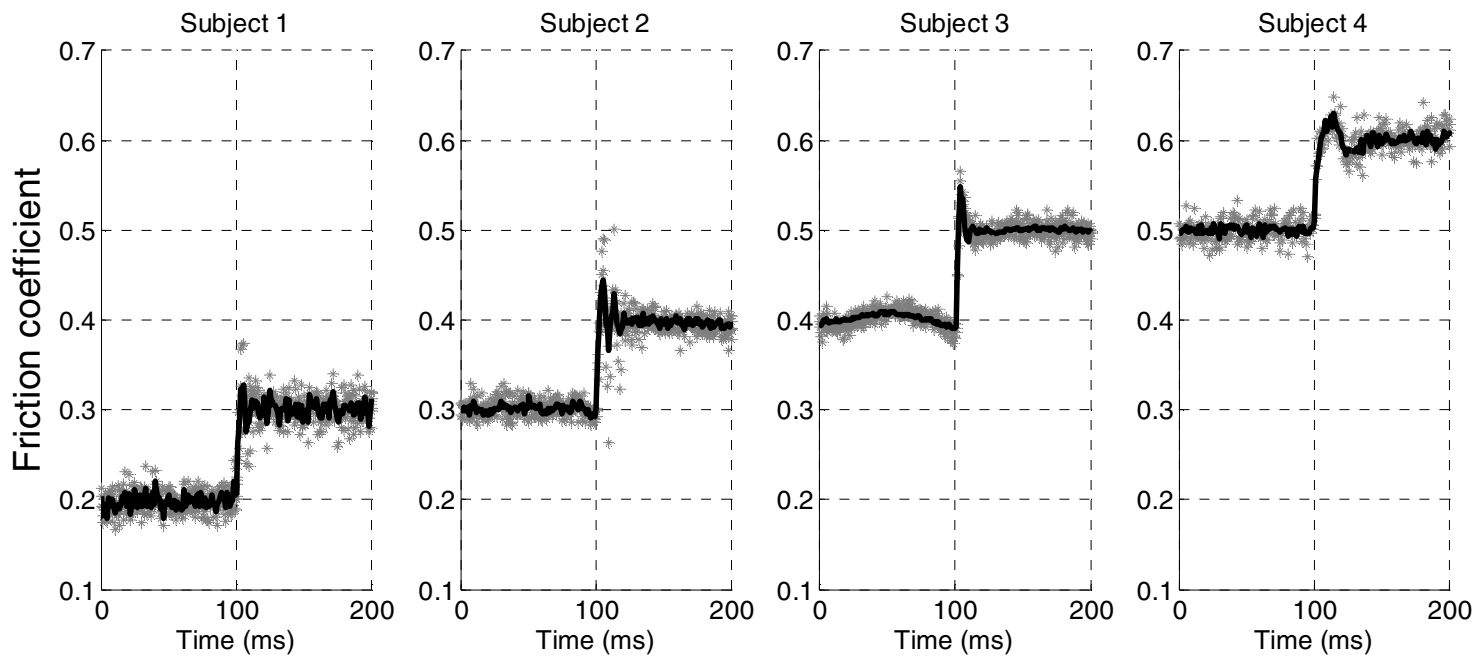

Fig. 7: Closed loop control of the friction coefficient as a function of time for the four participants. The dotted curves illustrate at least four try of touching the surface and the black continuous curve represents the average of all measurements for each subject.

When comparing the mean curve of the controlled friction for the 4 subjects, we find that the control strategy works well in static regime when imposing two references to the controller. In dynamic regime, there is a little difference when comparing the controlled friction for subjects; Tab. 1 shows a comparison in the dynamic regime of the rise time and the overshoot for each subjects. We find that the rise time is equal to $3.84 \pm 1.27 \mathrm{~ms}$ and the overshoot of $37.5 \pm 10.2 \%$.

Table I: The difference between the rise time and the overshoot between the four subjects.

\begin{tabular}{|l|c|c|c|c|c|}
\hline & Subject 1 & Subject 2 & Subject 3 & Subject 4 & Mean \\
\hline Rise time (ms) & 3.37 & 3.41 & 2.87 & 5.72 & 3.84 \\
\hline Overshoot (\%) & 27.6 & 44.4 & 48 & 30 & 37.5 \\
\hline
\end{tabular}




\section{Conclusion}

In this paper, we proposed a new high fidelity smart tactile stimulator called "SMARTTAC". An approach to control the wave amplitude of the vibration was presented. The objective is to make the effect of the tactile feedback phenomenon insensitive to external disturbances. For that aim, a new modelling has been developed analytically due to an accurate choice of the d-q frame. Identification has been performed and then, a simultaneous double closed loop control has been implemented. The tracking of the resonant shows a high robustness against the temperature variation. The capability of this tactile stimulator to control in real time the friction coefficient was also exposed by proposing a direct control of the friction thanks to the sensors implementation in the stimulator. Tribological validation of the COF control was proved by repeated experiment with four subjects showing a stable and desired level of friction with a low response time of about $4 \mathrm{~ms}$.

\section{References}

[1] T. Nara, M. Takasaki, T. Maeda, T. Higuchi, S. Ando, and S. Tachi, "Surface Acoustic Wave (SAW) tactile display based on properties of mechanoreceptors," in IEEE Virtual Reality, 2001. Proceedings, 2001, pp. 13-20.

[2] O. Bau, I. Poupyrev, A. Israr, and C. Harrison, "TeslaTouch: electrovibration for touch surfaces," in Proceedings of the 23nd annual ACM symposium on User interface software and technology, New York, NY, USA, 2010, pp. 283-292.

[3] E. Vezzoli, M. Amberg, F. Giraud, and B. Lemaire-Semail, "Electrovibration modelling analysis," in Eurohaptic 2014, Versailles, France, 2014.

[4] C. Winter, Y. Civet, and Y. Perriard, "Optimal design of a squeeze film actuator for friction feedback," in Electric Machines Drives Conference (IEMDC), 2013 IEEE International, 2013, pp. 1466-1470.

[5] T. Watanabe and S. Fukui, "A method for controlling tactile sensation of surface roughness using ultrasonic vibration," in IEEE International Conference on Robotics and Automation, 1995, vol. 1, pp. 1134-1139.

[6] P.-M. Lee, "Behavioral Model of Online Purchasers in E-Commerce Environment," Electron. Commer. Res., vol. 2, no. 1-2, pp. 75-85, Jan. 2002.

[7] M. Biet, F. Giraud, and B. Lemaire-Semail, "Implementation of tactile feedback by modifying the perceived friction,” Eur. Phys. J. - Appl. Phys., vol. 43, no. 01, pp. 123-135, 2008.

[8] L. Winfield, J. Glassmire, J. E. Colgate, and M. Peshkin, "T-PaD: Tactile Pattern Display through Variable Friction Reduction," in EuroHaptics Conference, 2007 and Symposium on Haptic Interfaces for Virtual Environment and Teleoperator Systems. World Haptics 2007. Second Joint, 2007, pp. 421-426.

[9] W. Ben Messaoud, B. Lemaire-Semail, M.-A. Bueno, M. Amberg, and F. Giraud, "Closed-Loop Control for Squeeze Film Effect in Tactile Stimulator," presented at the Proceedings of the 2014 international Conference and exhibition on new actuators and drives, Actuator 2014, Bremen, Germany, 2014, pp. 245 248.

[10] M. Amberg, F. Giraud, B. Semail, P. Olivo, G. Casiez, and N. Roussel, "STIMTAC: a tactile input device with programmable friction," in Proceedings of the 24th annual ACM symposium adjunct on User interface software and technology, New York, NY, USA, 2011, pp. 7-8.

[11] M. Wiertlewski, D. Leonardis, D. J. Meyer, M. A. Peshkin, and J. E. Colgate, "A High-Fidelity SurfaceHaptic Device for Texture Rendering on Bare Finger," in Haptics: Neuroscience, Devices, Modeling, and Applications, M. Auvray and C. Duriez, Eds. Springer Berlin Heidelberg, 2014, pp. 241-248.

[12] W. Leonhard, Control of Electrical Drives. Springer-Verlag, 1985.

[13] F. Pigache, F. Giraud, and B. Lemaire-Semail, "Modelling and identification of a planar standing wave ultrasonic motor," Eur. Phys. J. - Appl. Phys., vol. 34, no. 01, pp. 55-65, Apr. 2006.

[14] F. Giraud, M. Amberg, and B. Lemaire-Semail, "Design and control of a haptic knob," Sens. Actuators Phys., vol. 196, pp. 78-85, 2013.

[15] S. M. Pasumarty, S. A. Johnson, S. A. Watson, and M. J. Adams, "Friction of the Human Finger Pad: Influence of Moisture, Occlusion and Velocity," Tribol. Lett., vol. 44, no. 2, pp. 117-137, Nov. 2011.

[16] S. E. Tomlinson, R. Lewis, X. Liu, C. Texier, and M. J. Carré, "Understanding the Friction Mechanisms Between the Human Finger and Flat Contacting Surfaces in Moist Conditions," Tribol. Lett., vol. 41, no. 1, pp. 283-294, Jan. 2011.

[17] Y. Shtessel, C. Edwards, L. Fridman, and A. Levant, "Introduction: Intuitive Theory of Sliding Mode Control," in Sliding Mode Control and Observation, Springer New York, 2014, pp. 1-42.

[18] V. Utkin and H. Lee, "Chattering Problem in Sliding Mode Control Systems," in International Workshop on Variable Structure Systems, 2006. VSS'06, 2006, pp. 346-350. 